\title{
EXPERIENCE AND NEW APPROACH FOR REMOTE EDUCATION IN HETEROGENEOUS NETWORKS
}

\author{
E. DEL RE and L. PIERUCCI \\ University of Florence, Department of Electronic Engineering \\ Via S.Marta 3 \\ 50139 Firenze, ITALY
}

\begin{abstract}
The availability of broadband networks and the advent of standards and technologies for real time multi-point communications allow new educational systern. The paper describes a live remote Educational service in satellite/terrestrial environments and an Educational Tele-Medicine service in $\mathrm{Ku} / \mathrm{Ka}$ band.
\end{abstract}

\section{INTRODUCTION}

In a Remote Learning Service the users, the teacher and the students, not all at the same location make a virtual classroom. The teacher and the students must interact during the remote lessons as in a traditional one and can ask questions, exchange text, images or move the same pointer. Therefore, the classroom must be equipped differently to support remote education requirements: cameras show the teacher, the board and all the student in the classroom; the board can became a video projector which shows computer graphics/equation and all the didactic material necessary to the lecture; a communication link such as BRI ISDN or satellite is necessary to connect remote classrooms. Remote education uses audio, data and full-motion video according to different possible topologies:

- Live mode Any person anywhere can be telepresent in the class and participate in the lesson. The students can receive remote lectures, remote exercises or partecipate in remote to an experiment controlling the complete model of the events and selecting other measurement or signals to understand precisely what is happening. An educational service in real time, live and interactive way has to be offered.

- Archive mode The students can access to libraries archives, to virtual museums, to multimedia nagazines and to proper multimedia university courses or to multimedia didactic materials (CD-Rom or pre-recorded video or Web pages). The archival system could have a list of frequently asked questions (FAQ) by the students with the relative annotate answers as if the instructor were answering in real time. 
The requirements for an Interactive Remote Educational Service can be summarized in the following:

- Type of service: Multicasting/Unicasting

- Type of connection: Point-to-Multipoint in live mode and point-to-point in archive mode

- Level of interactivity: Medium to high, depending on the type of remote service

- Type of traffic: Symmetric transmission (Remote Lectures, Remote Exercise) or asymmetric (archive mode), continuous or bursty depending on type of remote service

- Type of multimedia data: Audio, Video, Data

- Throughput reqs. vs. type of data: Videoconference quality with at least $384 \mathrm{kbps}$ or more for a better quality of image and audio

The paper describes experience and innovative approaches followed to provide interactive multimedia Remote Education services via satellite environment. In particular it shows Live Remote Education realized in $\mathrm{Ka}$ band (work supported by the Italian Space Agency ASI and developed by the National Consortium on Telecommunications - CNIT) and a Tele- medicine education environment developed in the ACTS-ISIS (Interactive Satellite multimedia Information System) European Union project. The role of satellite must be regarded as integrated with the ground networks for its capability to connect quickly to networks locations otherwise geographically or economically unreachable. A new opportunity is provided by the satellite capacity to adapt the covered area to the changes in demands of services, in particular this is a good facility for multimedia services where, up to now, it is difficult to forecast different types of services and bit rates.

Moreover the broadcast transmission capability of satellite is very well suited to the interactive remote learning needs while, up to now, $\mathrm{r}$ remote learning on the Ethernet LAN/WAN is essentially a videoconference applications characterized by a point-to-point interaction. Therefore, it is important to investigate multimedia applications in heterogeneous networking environments where it can be seen with high interest the interconnection of IP/ATM networks via satellite. Across these various networks protocols may differ, link capacities may vary $(64 / 128 \mathrm{kbps}$ for ISDN, $2 \mathrm{Mbs}$ for satellite, $100 \mathrm{Mbps}$ for LANs, $>155$ Mbps to ATM networks), and it is necessary to overcame these difference to distribute multimedia information via multicast transmission (one-to-many or many-to-many) with guarantee Quality of Services, QoS. 


\section{LIVE REMOTE EDUCATION IN SATELLITE/TERRESTRIAL ENVIRONMENT}

In the ASI/CNIT project started this year, live lessons are delivered among several Italian Universities differently interconnected by ISDN to different ground stations in Ka band for ITALSAT II (initially in Florence, Bologna, Rome) as shown in fig.1. The use of Ka band allows the diffusion of multime-

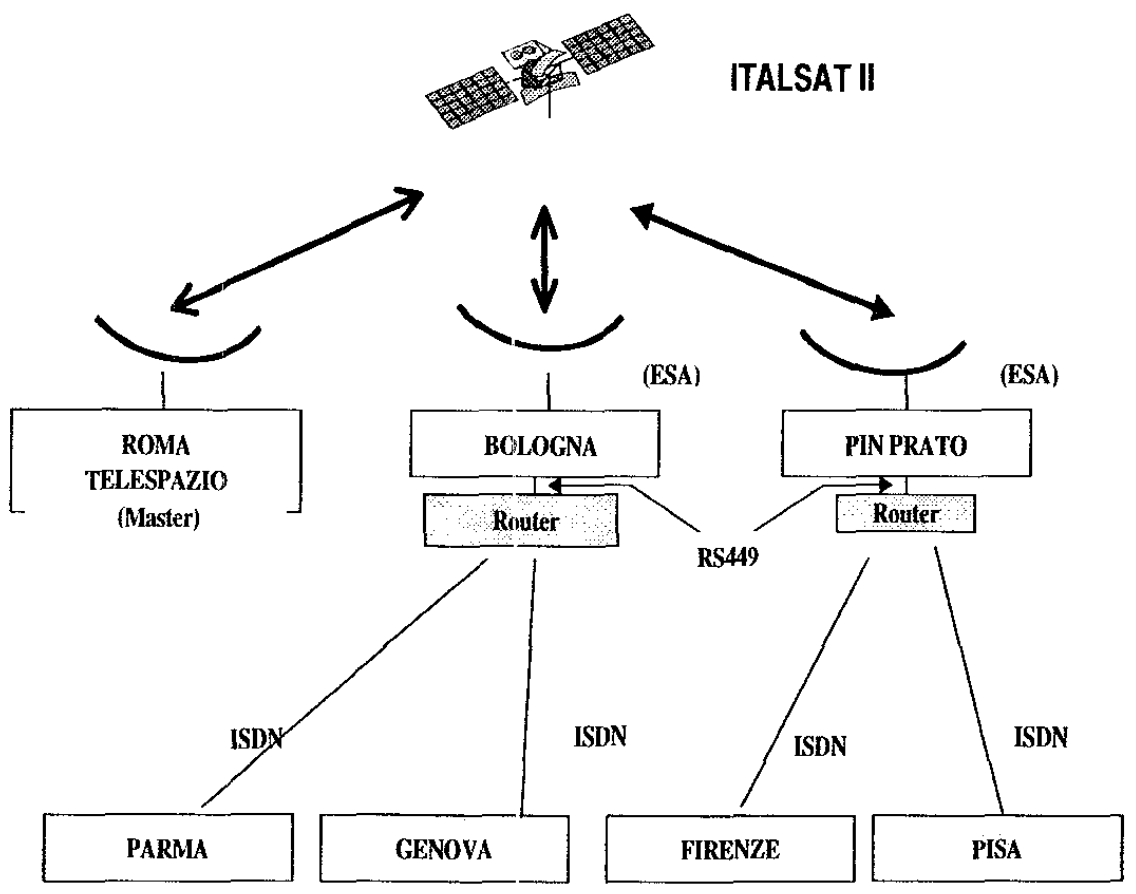

Figure 1: CNIT-ASI system topology

dia and interactive Remote Education services, due to the available wide-band capacity in the two directions (forward and return link) to reduce the size and weight of the user terminal. The ground stations for ITALSAT available from the CNR Telecommunications project, can establish two different channels among the user terminals, one at a rate of $384 \mathrm{~Kb} / \mathrm{s}$ for videoconferencing and one at a rate of $2-8 \mathrm{Mb} / \mathrm{s}$ for LAN internetworking. A slotted -aloha control channel permits a master terminal to dynamically assign satellite resources to the user terminals. A point-to-multipoint application delivers live lessons among many remote classrooms and according to a reservation list subsequently the students can interact with the teacher, asking information, 
using shared white board in real time.

Live lessons using this satellite plus terrestrial networks infrastructure, are analyzed in terms of BER, quality of images and other QoS. Subjective tests on quality of image (Visual impairment, interactivity, static quality, dynamic quality) according to ITU-R 500-5 are developed giving points from 1 to 5 . The following table presents the results obtained adding white noise on the received images to degrade the quality of image.

\begin{tabular}{|c||c|c|c|c|}
\hline Eb/No & Visual Impairments & Interactivity & Static Qual. & Dynamic Qual. \\
\hline \hline 3.5 & 1.75 & 2 & 1.5 & 1.12 \\
\hline 3.8 & 2.87 & 2.87 & 2.62 & 2 \\
\hline 4.1 & 3.75 & 3.12 & 3.12 & 2.37 \\
\hline$\infty$ & 4.12 & 3.37 & 4.25 & 3.5 \\
\hline
\end{tabular}

Multiple access protocols and bandwidth dynamic assignment, the impact of ATM technique on satellite are under investigation to provide an efficient Remote Education service.

\section{TELE-MEDICINE EDUCATION SERVICE IN KA/KU ENVIRONMENT}

The data collected in diagnostics images and video using $\mathrm{X}$ ray, magnetic resonance imaging, nuclear medicine provides an important reference and diagnostic tool for physicians. To medical training purposes a medical reference images data base is important to get practice in distinguish between normal and abnormal situations.

The Tele-medicine application MEDI (Medical Environment for Diagnostic Images) developed into the ACTS-ISIS European project, permits the access to a reference database of medical diagnostic images and related image processing in order to medical training. A student can retrieve an image using Web pages with many indication of the clinical case and finally he has the possibility to invite one or more experienced physicians for detailed information about the sclected case and providing links to more in-depth descriptions. The fig. 2 shows an example of MEDI tool. The images of relation data base are in Papyrus 3.0 format in agreement with DICOM 3.0 part 10 standard and the Java Database Connectivity (JDBC) is used by the JAVA applet to send SQL statements and access to the relational data base.

MEDI is a WEB application based on HTML pages, JAVA client front end (applets) and JAVA servers and has an open architecture with the following characteristics:

- it has been developed in JAVA language, therefore it is machine independent 
- it uses the TCP/IP protocol, so it can run on different type of networks

- it is independent from the commercial browser used

- it is particularly suitable so broadcast connections for its clients/server architecture.

The ISIS project, a program commissioned by the European Commission DGXIII within the ACTS 4th Framework Program, develops a satellite communications system based on dual band concept and suitable to provide interactive multimedia services.

The basic ISIS architecture is formed by a Service Center that provides all feeder links functions and the storage of data images and videos, and different user-stations typically the user $\mathrm{PC}$, equipped with the dual-band terminal, $\mathrm{Ku}$ frequency band (EUTELSAT) for a high rate forward link and Ka frequency band (ITALSAT II) for the return link, at low- cost, with small size, easily transportable and installed.

In this configuration the MEDI server (Java servers and HTTP server, medical reference data base) located at the Service Center, is connected to the client via EUTELSAT satellite (forward channel) and the link from the client to the server via ITALSAT satellite (return channel) using the ISIS platform. The client equipped with dualband terminal able to receive and transmit multimedia signals can start and see the MEDI application using a commercial browser such as Netscape, Explorer or HotJava. A number of tests (ping, FTP, HTTP) are carried out to validate the ISIS platform as in the following table 1

\begin{tabular}{|c||c|}
\hline Test & Results \\
\hline PING & Connectivity OK \\
& Round Trip Times: $559-800 \mathrm{~ms}$ \\
& Packet loss: $0,014 \%$ \\
\hline FTP & Connectivity OK \\
& $\begin{array}{c}\text { Successfu } 10 \mathrm{k}-100 \mathrm{k}-1000 \mathrm{k} \text { file transfer OK } \\
>10 \mathrm{Kbyte} / \mathrm{s} \text { throuhput for one session } \\
\end{array}$ \\
\hline HTTP & No packets loss \\
\hline
\end{tabular}

Table 1: Network tests

The table 2 shows the transfer timing of image of different size from the server to client.

On line information about the MEDI application can be retrieved on the site http:// amerigo.die.unifi.it/isis/index.html 


\begin{tabular}{||c||c||c||}
\hline \multicolumn{1}{|c||}{} & Transfer of image & Validation Criteria \\
\hline \hline$X-$ ray $135 k B$ & $80^{\prime \prime}$ & Shared tools OK \\
\hline Cinecard $2 M B$ & $25^{\prime}$ & Movie OK Shared tools OK \\
\hline Magnetic Resonance $1 M B$ & $12^{\prime}$ & Shared tools OK \\
\hline
\end{tabular}

Table 2: Timing transfer on forward link

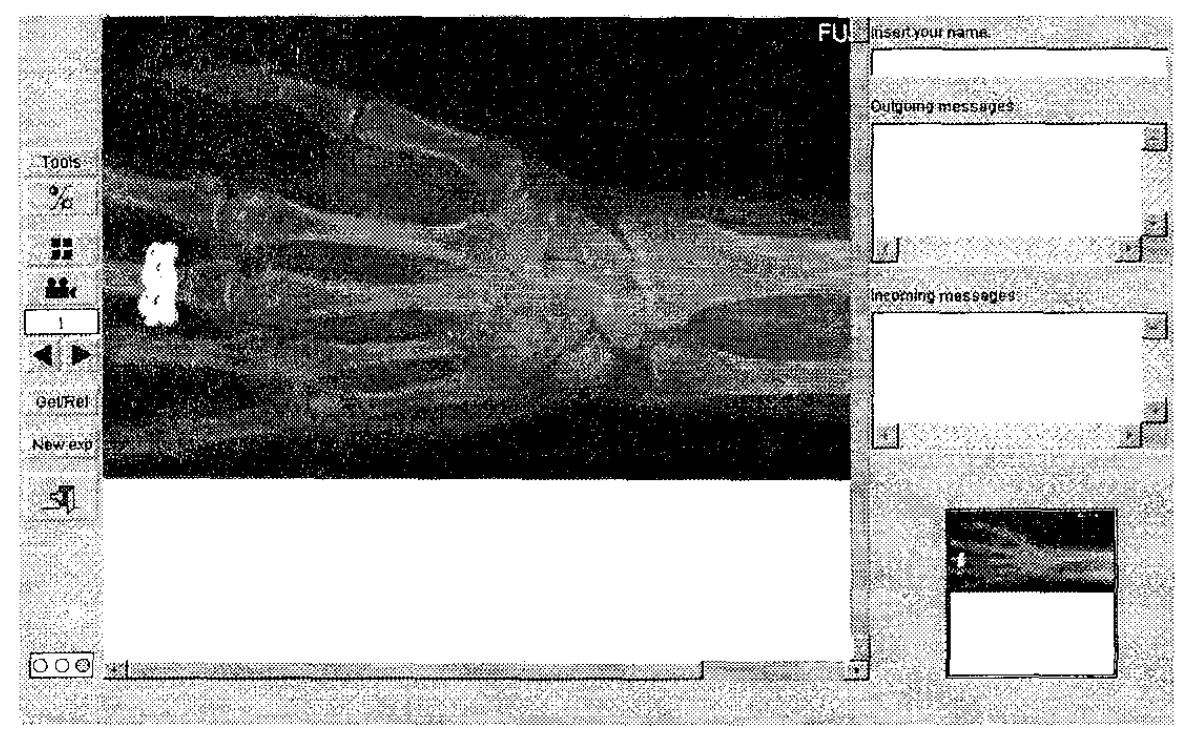

Figure 2: MEDI tools

\section{ACKNOWLEDGMENTS}

The authors wish to thank the ACTS-ISIS Consortium members and the Italian Space Agency to support the work.

\section{References}

[1] B.Barcelo, J. A. Inkrott, A. R. Snowdon, K. J. Trojniar "A multimedia Distance Learning Trial Using ISDN BRI",ATETT TECHNICAL JOURNAL January/February 1993.

[2] A.Banerjea "Heterogeneous Networking",IEEE Multimedia April-June 1997. 
[3] "Technical Annex" ISIS Projects AC103, ISIS Projects Deliverables, AC103/NTLZ/IIS/DS/019/a1; AC103/NTLZ/IIS/DS/018/a1;AC103/NTLZ/IIS/DS/005/a1; AC103/NTLZ/IIS/DS/022/a1 1996-1998. 